\title{
Tratamiento de Strongyloides stercoralis con ivermectina y tiabendazole
}

\author{
PEDRO HUAPAYA ${ }^{1,2}$, YRMA ESPINOZA ${ }^{1,2}$, ALINA HUIZA ${ }^{1,2}$, \\ CARLOS SEVILLA ${ }^{2}$, HERMAN VILDÓSOLA ${ }^{1}$ \\ ${ }^{\prime}$ Instituto de Medicina Tropical "Daniel A. Carrión”, UNMSM. \\ ${ }^{2}$ Departamento Académico de Microbiología Médica, Facultad de Medicina, UNMSM.
}

\begin{abstract}
RESUMEN
OBJETIVO: Describir la experiencia del uso de ivermectina y tiabendazole en pacientes atendidos en el Instituto de Medicina Tropical "Daniel A. Carrión”- UNMSM. MATERIAL Y MÉTODOS: Durante los años 2001 y 2002, se administró ivermectina $0,2 \mathrm{mg} / \mathrm{kg}$ en dosis única a 22 pacientes (Grupo 1) o tiabendazole $25 \mathrm{mg} / \mathrm{kg}$ por 3 días a 20 pacientes (Grupo 2) con diagnóstico de Strongyloides stercoralis en heces. Se efectuó controles entre 20 y 40 días después de administrado el tratamiento. RESULTADOS: El promedio de edades fue 21,8 años (DE 22,6) para el grupo 1 y de 33,5 años (DE 14,2) para el grupo 2. Hubo 12 varones $(54,5 \%)$ en el grupo 1 y 7 (35\%) en el grupo 2. Ivermectina fue $100 \%$ eficaz, mientras que tiabendazole lo fue en $95 \%$; sólo un caso requirió un segundo ciclo debido a la alta carga parasitaria inicial; el siguiente control fue negativo. Los síntomas más frecuentes fueron diarreas $(71,4 \%)$, dolor cólico $(61,9 \%)$ y dolor epigástrico $(47,6 \%)$. Todos los pacientes manifestaron mejoría clínica luego del tratamiento. Sólo 2 casos (10\%) del grupo 2 manifestaron leve sensación nauseosa durante el primer día del tratamiento, que remitió por completo al segundo día. CONCLUSIONES: Ambos medicamentos ratifican su eficacia y seguridad para ser utilizados en el tratamiento de Strongyloides stercoralis; es necesario disponer de ellos en el petitorio nacional.
\end{abstract}

Palabras clave: Estrongiloidiasis; ivermectina; Strongyloides stercoralis; antihelminticos.

\section{TREATMENT OF Strongyloides stercoralis WITH IVERMECTIN AND THIABENDAZOLE SUMMARY}

OBJECTIVE: To describe the experience with ivermectin and thiabendazole in patients attended at the Tropical Medicine Institute "Daniel A. Carrión"- UNMSM. MATERIAL AND METHODS: During 2001 and 2002, ivermectin $(0,2 \mathrm{mg} / \mathrm{kg}$ in single dosis) and thiabendazole ( $25 \mathrm{mg} / \mathrm{kg} / 3 \mathrm{days})$ were administered to 22 (Group 1) and 20 (Group 2) patients with diagnosis of Strongyloides stercoralis in stools. Controls were performed between 20 and 40 days following treatment. RESULTS: Mean age was 21,8 years (SD 22,6) for group 1 and 33,5 years (SD 14,2) for group 2. There were 12 males $(54,5 \%)$ in group 1 and 7 (35\%) in group 2 . Ivermectin was $100 \%$ efficient while thiabendazole was $95 \%$; only one case needed a second cycle because of high amount of parasites from the beginning; his next control was negative. Most frequent symptoms were diarrhea $(71,4 \%)$, abdominal pain $(61,9 \%)$ and epigastric pain $(47,6 \%)$; all patients felt better after treatment. Only 2 cases (10\%) from group 2 referred light nausea during the first day of treatment that disappeared at the second day. CONCLUSIONS: Both drugs ratify their efficacy and safety for Strongyloides stercoralis treatment making it necessary to have them in the national petitioner.

Key words: Strongyloidiasis; ivermectin; Strongyloides stercoralis; anthelmintics.

\footnotetext{
Correspondencia:

Dr. Pedro Ernesto Huapaya Herreros

Instituto de Medicina Tropical "Daniel A. Carrión"

UNMSM - Sección de Parasitología.

Jr. José Santos Chocano 199. Urb. San Joaquín

Bellavista, Callao 02

E-mail:pehh@terramail.com.pe
} 


\section{INTRODUCCIÓN}

La infección causada por el nemátode Strongyloides stercoralis tiene en el Perú relativa frecuencia en la población, especialmente en la selva $\left(^{1-6}\right)$. Ocasiona diversos problemas, como diarreas, dolor epigástrico, pérdida de peso, anemia e inclusive el peligroso síndrome de hiperinfección, que muchas veces puede llegar a ser mortal debido a las múltiples complicaciones que puede originar $\left(^{7-16}\right)$. No se dispone en nuestro país de un esquema de tratamiento totalmente eficaz que permita el manejo de esta infección en forma oportuna para evitar complicaciones.

Es conocido que existen dos drogas altamente eficaces contra este parásito, ivermectina $\left({ }^{13,17-21}\right)$ y tiabendazole $\left({ }^{22-26}\right)$, lo que las convierte en drogas de primera elección para el manejo de estos casos. Sin embargo, por razones no bien explicadas, ivermectina no circula en el mercado farmacéutico nacional y tiabendazole existe pero en forma restringida, sin ser accesible a la población que padece el problema. Ambos medicamentos se encuentran disponibles en centros de investigación, como el Instituto de Medicina Tropical "Daniel A. Carrión" de la UNMSM, donde se les utiliza en forma cotidiana y se brinda gratuitamente al público que concurre para buscar atención.

Es importante describir periódicamente la experiencia que existe en nuestro país con estos medicamentos, ya que los demás fármacos antiparasitarios utilizados comúnmente en nuestro país no ofrecen toda la eficacia y seguridad deseadas para estos casos $\left({ }^{1,13,17}\right)$. Este trabajo describe los resultados en nuestra institución, para así promover el ingreso de ambos medicamentos al mercado nacional y enfrentar mejor esta infección.

\section{MATERIAL Y MÉTODOS}

Durante los años 2001 y 2002, en el Instituto de Medicina Tropical "Daniel A. Carrión" de la Universidad Nacional Mayor de San Marcos se detectó casos de infección intestinal por Strongyloides stercoralis, tanto en población residente en Lima como en otras ciudades. Las personas acudieron al consultorio externo de nuestra institución o fueron derivados de otros establecimientos y/o fueron detectados mediante la ejecución de actividades de detección masiva de enteroparásitos en la población de Lima y del interior del país.

Las personas infectadas fueron atendidas por el personal de la Sección de Parasitología, para la evaluación clínica y el tratamiento farmacológico correspondiente.

La evaluación clínica incluyó entrevista médica y examen general, con énfasis en las molestias que los pacientes manifestaron. Asimismo, se registró los datos de filiación y antecedentes de importancia (lugar de nacimiento, visitas a la selva). También se explicó la razón de la administración gratuita de esos medicamentos.

Los fármacos administrados fueron ivermectina y tiabendazole, ambos disponibles en distintos momentos, debido a donaciones de laboratorios farmacéuticos o de otras instituciones que gentilmente los brindaron a nuestro establecimiento, con el fin de facilitar la terapia de esta infección, ante el conocimiento de la dificultad de encontrarlos en el mercado nacional.

Los tratamientos fueron administrados a dosis convencionales, es decir, ivermectina a 0,2 $\mathrm{mg} / \mathrm{kg}$ en dosis única y tiabendazole a razón de $25 \mathrm{mg} / \mathrm{kg} /$ día durante 3 días. Los fármacos no fueron administrados en forma aleatoria; dependió de la disponibilidad de los medicamentos en cuanto a cantidad y oportunidad. Principalmente se trató de administrar tiabendazole a adultos e ivermectina a menores de edad, debido a los reportes de las reacciones adversas que podía ocasionar el primero $\left({ }^{27-29}\right)$; sin embargo, esto no fue un criterio absoluto.

Los casos fueron detectados utilizando diversos métodos de examen parasitológico, principalmente examen directo seriado, sedimentación rápida y la técnica de Baermann. El control 
parasitológico se realizó entre los 20 y 40 días después de haber concluido el tratamiento; ello dependió de la accesibilidad de los pacientes al establecimiento o viceversa, ya que algunos de ellos fueron detectados y controlados fuera de Lima.

Los controles se realizaron mediante examen seriado de heces (3 muestras), las cuales fueron examinadas mediante las técnicas ya descritas, y algunos fueron complementados con el cultivo en carbón vegetal. Asimismo, se repitió la evaluación clínica para evidenciar la mejoría de cada persona.

\section{RESULTADOS}

Durante los años 2001 y 2002, se detectó un total de 42 casos de estrongiloidiasis; 21 casos fueron detectados en el Instituto y los otros 21 en el distrito de Perené, provincia de Chanchamayo, Junín, durante una actividad extramural del personal de la Sección de Parasitología.

De los 21 casos detectados en Lima, 18 $(85,7 \%)$ refirieron visitas o residencia en ciudades de la selva endémicas al parásito, $2(9,5 \%)$ no tenían este antecedente, pero sí algún familiar cercano (cónyuge, hermanos, hijos), y sólo 1 caso $(4,8 \%)$ no manifestó antecedente de este tipo.

Los clasificamos en dos grupos según el tratamiento recibido; el grupo 1 (22 casos) recibió ivermectina y el grupo 2 (20 casos), tiabendazole, a las dosis ya indicadas.

Los promedios de edades fueron 21,8 años (DE 22,6) para el grupo 1 y 33,5 años (DE 14,2) para el grupo 2 (Tabla 1). Hubo 12 varones $(54,5 \%)$ en el grupo 1 y $7(35 \%)$ en el grupo 2 (Tabla 2).

Ivermectina fue $100 \%$ eficaz, mientras que tiabendazole lo fue en $95 \%$; sólo un caso requirió un segundo ciclo de tratamiento, debido a la alta carga parasitaria que presentó al inicio:
Tabla 1.- Distribución por edades de la población estudiada.

\begin{tabular}{|c|c|c|c|c|c|c|}
\hline \multirow[t]{2}{*}{ Edad (años) } & \multicolumn{2}{|c|}{ Grupo 1} & \multicolumn{2}{|c|}{ Grupo 2} & \multicolumn{2}{|c|}{ Total } \\
\hline & $\mathrm{n}$ & $\%$ & $\mathrm{n}$ & $\%$ & $\mathrm{n}$ & $\%$ \\
\hline 1 a 4 & 5 & 22,7 & 0 & 0,0 & 5 & 11,9 \\
\hline 5 a 14 & 7 & 31,8 & 2 & 10,0 & 9 & 21,4 \\
\hline 15 a 44 & 6 & 27,3 & 14 & 70,0 & 20 & 47,6 \\
\hline 45 a 60 & 2 & 9,1 & 3 & 15,0 & 5 & 11,9 \\
\hline Más de 60 & 2 & 9,1 & 1 & 5,0 & 3 & 7,1 \\
\hline Total & 22 & 100,0 & 20 & 100,0 & 42 & 100,0 \\
\hline Promedio & \multicolumn{2}{|r|}{21,8} & \multicolumn{2}{|c|}{33,5} & & \\
\hline $\mathrm{DE}$ & \multicolumn{2}{|r|}{22,6} & \multicolumn{2}{|c|}{14,2} & & \\
\hline Edad mínima & \multicolumn{2}{|c|}{2} & \multicolumn{2}{|c|}{14} & & \\
\hline Edad máxima & \multicolumn{2}{|r|}{70} & \multicolumn{2}{|c|}{62} & & \\
\hline
\end{tabular}

aproximadamente se contó más de 400 larvas en un extendido de heces en una lámina cubreobjeto de $22 \times 22 \mathrm{~mm}$, siendo negativo en el control después del segundo tratamiento. La carga parasitaria de la mayoría de los demás casos no llegaba a 50 en la misma preparación.

Los síntomas más frecuentes fueron diarrea $(71,4 \%)$, de olor fétido, abundante, sin moco ni sangre, sin pujo ni tenesmo. Dolor tipo cólico $(61,9 \%)$ sin localización definida, aunque algunos pacientes refirieron el flanco derecho como el sitio más frecuente; dolor epigástrico $(47,6 \%)$, descrito como ardor, de preferencia posprandial, asociado a dispepsia, a pesar de haber recibido tratamiento previo con antiácidos diversos.

También manifestaron aumento de ruidos hidroaéreos $(40,5 \%)$, descrito como borbo-

Tabla 2.- Distribución por sexos.

\begin{tabular}{|c|c|c|c|c|c|c|}
\hline \multirow[t]{2}{*}{ Sexo } & \multicolumn{2}{|c|}{ Grupo 1} & \multicolumn{2}{|c|}{ Grupo 2} & \multicolumn{2}{|c|}{ Total } \\
\hline & $\mathrm{n}$ & $\%$ & $\mathrm{n}$ & $\%$ & $\mathrm{n}$ & $\%$ \\
\hline Masculino & 12 & 54,5 & 7 & 35,0 & 19 & 45,2 \\
\hline Femenino & 10 & 45,5 & 13 & 65,0 & 23 & 54,8 \\
\hline Total & 22 & 100,0 & 20 & 100,0 & 42 & 100,0 \\
\hline
\end{tabular}


rismos, meteorismo, flatulencia; náusea y/o vómitos $(16,7 \%)$ y estreñimiento $(7,1 \%)$ (Tabla 3). Todos los pacientes manifestaron mejoría clínica progresiva luego del tratamiento; inclusive el caso que requirió segundo tratamiento mejoró de sus molestias después del primer ciclo, siendo la sintomatología escasa luego del segundo tratamiento.

Tabla 3.- Manifestaciones clínicas iniciales.

\begin{tabular}{|c|c|c|c|c|c|c|}
\hline \multirow[t]{2}{*}{ Clínica } & \multicolumn{2}{|c|}{ Grupo 1} & \multicolumn{2}{|c|}{ Grupo 2} & \multicolumn{2}{|c|}{ Total } \\
\hline & $\mathrm{n}$ & $\%$ & $\mathrm{n}$ & $\%$ & $\mathrm{n}$ & $\%$ \\
\hline Diarreas & 15 & 68,2 & 15 & 75,0 & 30 & 71,4 \\
\hline Dolor cólico & 11 & 50,0 & 15 & 75,0 & 26 & 61,9 \\
\hline Epigastralgia & 7 & 31,8 & 13 & 65,0 & 20 & 47,6 \\
\hline RHA & 9 & 40,9 & 8 & 40,0 & 17 & 40,5 \\
\hline Náuseas, vómitos & 4 & 18,2 & 3 & 15,0 & 7 & 16,7 \\
\hline Estreñimiento & 0 & 0,0 & 3 & 15,0 & 3 & 7,1 \\
\hline Total & 22 & 100,0 & 20 & 100,0 & 42 & 100,0 \\
\hline
\end{tabular}

No se presentaron reacciones adversas en el grupo 1 , mientras que sólo 2 casos $(10 \%)$ del grupo 2 manifestaron leve sensación nauseosa durante el primer día del tratamiento, que remitió por completo al segundo día y no requirió modificar el esquema ni tratamiento sintomático adicional.

\section{DISCUSIÓN}

Los resultados de la experiencia del uso de estos fármacos concuerdan con los reportes internacionales de eficacia de ambas drogas $(8,13,17,18,20-25)$, la cual se mantiene a menos que existan otros problemas de salud concomitantes ( $\left.{ }^{30-33}\right)$; así como también la seguridad que representan, especialmente tiabendazole, que según varios reportes es causante de reacciones adversas indeseables $\left({ }^{27-29}\right)$; ninguna de ellas se presentó en el grupo estudiado. En el caso de ivermectina, es sabido que es una droga muy segura con casi nula presentación de efectos indeseables $\left({ }^{17-21}\right)$.
Ante estas evidencias, es importante llamar la atención de las autoridades de salud para gestionar el ingreso de estos medicamentos al petitorio farmacológico nacional, a fin de que estén al alcance de las personas con esta infección y se pueda brindar el tratamiento de primera línea en forma oportuna.

En conclusión, tanto ivermectina como tiabendazole son drogas de alta eficacia para el tratamiento de infección por Strongyloides stercoralis. Ambas drogas son seguras cuando se las utiliza a las dosis recomendadas. Es importante considerar la necesidad del ingreso de ambos fármacos al mercado nacional.

\section{BIBLIOGRAFÍA}

1. Vildósola, G. Estrongiloidiasis. Rev Gastroenterol Perú 1997; 17, Supl 1: S75-S91.

2. Huapaya $P$, Chávez V, Valverde $S$, Rose M. Prevalencia de Strongyloidiasis en lactantes y pre-escolares de la Provincia de Chanchamayo. Bol Peruano Parasitol 1995; 11: 32-4.

3. Hernández F. Strongyloides stercoralis: un parásito subestimado. Parasitología al Día 2001; 25: 40-9.

4. Liñan-Abanto R, Jara C. Frecuencia y aspectos epidemiológicos del parasitismo por helmintos intestinales en la población infantil de Paiján, La Libertad-Perú. Bol Peruano Parasitol 1995; 11: 46-50.

5. Vásquez E, Gamero F, Aguirre E. Enteroparasitosis en el asentamiento humano Enrique Milla Ochoa - Los Olivos. Bol Peruano Parasitol 1995; 11: 55-6.

6. Tantaleán M, Atencia G. Nota sobre parasitismo intestinal diagnosticado en el IMT Daniel A. Carrión. Rev Per Med Trop UNMSM 1993; 7(2): 99-103.

7. Bannon J, Fater M. Intestinal ileus secondary to Strongyloides stercoralis infection: case report and review of the literature. Am Surgeon 1995; 61(4): 377-41.

8. Boyd W, Campbell F, Trudeau W. Strongyloides stercoralis - Hyperinfection. Am J Trop Med Hyg 1978; 27(1): 39-41.

9. Gotuzzo E, Terashima A, Alvarez H, Tello R, Infante R, Watts D, Freedman D. Strongyloides stercoralis hyperinfection associated with human $\mathrm{T}$ cell lymphotropic virus type -1 in Peru. Am J Trop Med Hyg 1999; 60(1): 146-9.

10. Goyal S. Intestinal strongyloidiasis manifesting as eosinophilic pleural effusion. Southern Med J 1998; 91 (8): 768-70.

11. Herrera J, Herrera J. Estrongiloidiasis: Presentación quirúrgica de dos casos en el Hospital Central SSPNP - 1988. Rev Sanidad Policía Nac 1989; 50(2): 110-8. 
12. Marchesan M, Cardoso R, Anefalos A, Kobayasi S. Invasive enteritis by Strongyloides stercoralis presenting as acute abdominal distress under corticosteroid therapy. Rev Hosp Clín Fac Med Sao Paulo 2001; 56 (4): 103-6.

13. Neva F. Biology and immunology of human strongyloidiasis. J Infect Dis 1986; 154(3): 397- 406.

14. Nonaka D, Takaki K, Tanaka M, Umeno M, Takeda T, Yoshida M, et al. Paralitic ileus due to Strongyloidiasis: case report and review of the literature. Am J Trop Med Hyg 1998; 59(4): 535-8.

15. Shelhamer J, Neva F, Finn D. Persistent Strongyloidiasis in an immunodeficient patient. Am J Trop Med Hyg 1982; 31(4): 746-51.

16. Terashima A, Gotuzzo E, Alvarez H, Infante R, Tello R, Watts D, Freedman D. Strongyloides stercoralis: Formas clínicas severas asociadas a infección por HTLV-I. Rev Gastroenterol Perú 1999; 19(1): 35-40.

17. Datry A, Hilmarsdottir I, Mayorga R, Lyagoubi M, Gaxotte P, Biligui S, et al. Treatment of Strongyloides stercoralis infections with ivermectin compared with albendazol: results of an open study of 60 cases. Transact Royal Soc Trop Med Hyg 1994; 88: 344-5.

18. Gann P, Neva F, Gam A. A randomized trial of single and two dose ivermectin versus thiabendazole for treatment of Strongyloidiasis. J Infect Dis 1994; 169: 1076-9.

19. Lindo J, Atkins N, Lee M, Robinson R, Bundy D. Parasite-specific serum IgG following successful treatment of endemic strongyloidiasis using ivermectin. Transact Royal Soc Trop Med Hyg 1996; 90: 702-3.

20. Náquira C, Jimenez G, Guerra J, Bernal R, Nalin D, Neu D, Aziz M. Ivermectin for human strongyloidiasis and other intestinal helminths. Am J Trop Med Hyg 1989; 40(3): 304-9.

21. Shikiya K, Kinjo N, Uehara T, Uechi H, Ohshiro J, Arakaki T, et al. Efficacy of ivermectin against Strongyloides stercoralis in humans. Internal Med 1993; 31(3): 310-2.

22. Most H, Yoeli M, Campbell W, Cuckler A. The treatment of Strongyloides and Enterobius infections with thiabendazole. Am J Trop Med Hyg 1965; 14(3): 37982.

23. Cahill K. Thiabendazol in massive strongyloidiasis. Am J Trop Med Hyg 1967; 16(4): 451-3.

24. Franz K, Schneider, PohIman M. Clinical trials with thiabendazole against intestinal nematodes infecting humans. Am J Trop Med Hyg 1965; 14(3): 383-6.

25. Grove D. Treatment of strongyloidiasis with thiabendazole: an analysis of toxicity and effectiveness. Transact Royal Soc Trop Med Hyg 1982; 76(1): 114-8.

26. Portugal R, Schaffel R, Almeida L, Spector N, Nucci M. Thiabendazole for the prophylaxis of strongyloidiasis in immunosuppressed patients with hematological diseases: a randomized double-blind placebo-controlled study. Haematol 2002; 87(6): 663-4.

27. Eland IA, Kerkhof SC, Overbosch D, Wismans PJ, Stricker BH. Cholestatic hepatitis ascribed to the use of thiabendazole. Ned Tijdschr Geneeskd 1998; 142(23): 1331-4.

28. Marron Gasca J, Gimenez Serrano H, Calvo Morales A, Pastor Bajo JA, Aizpun Pon M. Fixed exanthema induced by thiabendazole. Actas Dermosifiliogr 1976; 67(910): 701-6.

29. Tada Y, Fujitani T, Yano N, Yuzawa K, Nagasawa A, Aoki N, et al. Chronic toxicity of thiabendazole (TBZ) in CD-1 mice. Toxicol 2001; 169(3): 163-76.

30. Terashima A, Alvarez H, Tello R, Infante R, Freedman DO, Gotuzzo E. Treatment failure in intestinal Strongyloidiasis: an indicator of HTLV-1 infection. Int J Infect Dis 2002; 6(1): 28-30.

31. Davidson, R, Fletcher R, Chapman L. Risk factors for Strongyloidiasis. A case-control study. Arch Intern Med 1984; 144: 321-4.

32. Godoy P, Camargos C, Costa G, Fonseca L. Associação timoma e strongiloidíase intestinal grave. Rev Soc Brasileira Med Trop 1998; 31(5): 481-5.

33. Maciel E, Espinheira L, Brites C. Strongyloidiasis as an opportunistic infection in a HAM/TSP patient. Brazilian J Infect Dis 1999; 3(1): 23-7. 